\title{
MarconiNet supporting Streaming Media over Localized Wireless Multicast
}

\author{
Ashutosh Dutta, Subir Das, Anthony McAuley, Wai Chen \\ Telcordia Technologies Inc., 445 South Street, Morristown, NJ 07960 \\ Henning Schulzrinne \\ Computer Science Department, Columbia University, New York, NY 10027 \\ Onur Altintas \\ Toyota InfoTechnology Center, 4009 Miranda Avenue, Palo Alto, CA 94304
}

\begin{abstract}
-
Flexible multi-media streaming such as advertisment insertion, location based services, mobility and wireless access are vital components that make existing Internet Radio and TV networks more attractive for the roaming users. All of these applications also provide added value to telematics, and military usage including coordination, education, situation awareness, distributed simulation, battlefield communication and multi-player games. While content distribution over a wired network can be realized by instituting proxies and gateways at several parts of the access network, providing mobility over heterogeneous wireless access need to consider many operational issues such as handoff, join and leave latency and desired level of quality of service for the mobile clients. This paper discusses some novel application layer techniques that provide a platform for Mobile E-Commerce with a multi-tiered payment and security scheme that supports a business model for a global streaming network. The proposed streaming network called MarconiNet is based on standard IETF protocols such as SIP, SAP and SDP for signaling, RTSP for stream control and RTP/RTCP for media delivery and feedback control.
\end{abstract}

\section{INTRODUCTION}

Lately, streaming real-time multimedia content over the Internet is gaining momentum in the communications, entertainment, music and interactive game industries. Streaming application include IP telephony, broadcasting multimedia content, multi-party conferences, collaborations and multiplayer games. All of these applications would also have corresponding uses in a military context, including coordination, education, situation awareness, distributed simulation and battlefield communication. Real-time streaming content (audio and video) is mostly RTP/UDP based application which has stringent delay and loss requirements. Mobility however affects the delay and transient loss for the multimedia stream delivery to a great extent, because of associated continuous handoff. Thus it is desirable to be able to maintain continuity and provide proper QoS while moving between the cells in a wireless environment.

In order to make efficient use of network bandwidth within the core of the network, IP multicasting is used for wide area networking. There are several proposed scheme to provide native IP multicast routing over a wide area network such as PIM [1], MOSPF [2], DVMRP [3], CBT [4], BGMP [5]. However these suffer from lack of wide deployment since there are many issues such as pricing, QoS, maintenance of the router states in the core of the network. Recently there are however alternate techniques being developed such as Source Specific Multicast (SSM) [6], UMTP (UDP Multicast Tunneling Protocol) [7] and AMT (Automatic Multicast Tunneling Protocol) [8] that provide multicast support for the nonmulticast enabled networks while providing novel ways to support specific application such as content distribution. Reference [9] explains many of the issues associated with multicast deployment over wide area networking.

Localized Multicasting becomes more attractive for the mobile users experiencing intra-domain hand-off because of its ease of deployment and its ability to provide more flexible services such as localized advertisement, news broadcast, and location specific information in the wireless environment.

MarconiNet [10] proposes an integrated streaming architecture to support multimedia applications such as IP Telephony and broadcasting streaming content over the Internet. In this architecture the end nodes could be mobile and moving between access points, where the access points may belong to different cells, subnets and domains. As the end hosts move from one server to another server, it is desirable that the clients would still need to be part of the same multimedia stream while receiving the desired quality of service. Key factors that need to be taken into account however are; how quickly the end node leaves and joins the old and new multicast address space respectively, and how the quality of service is guaranteed as the end node moves from one cell to another.

Here we describe some of the scenario for wireless streaming multimedia using localized multicast that can be offered under MarconiNet environment, analyze the common latency factors involved and discuss various application layer approaches to take care of fast delivery of multicast stream and provide proper quality of service, as the end client moves and gets served by the local stations (servers) within a base station's area.

This paper is organized as follows. Section II gives a brief overview of MarconiNet. Section III cites some of the related work. Section IV outlines a business model viable for M-Commerce. Section V discusses the mobility components and various factors involved for fast-handoff. Section VI de- 
tails the possible alternatives to take care of seamless delivery of localized multicast stream in MarconiNet environment. Section VII briefly describes how QoS can be maintained for the mobile users under this scheme. Finally section VIII concludes the paper with open issues and future work.

\section{MARCONiNet OVERVIEW}

MarconiNet [10], [11] describes a multimedia streaming architecture that provides a way of content distribution over the Mobile Internet in a scalable way. As illustrated in the figure 2, there are 4 basic components in the MarconiNet architecture, namely Global Station (Primary Station), Radio Antenna Server (RAS) which are the local servers and Internet Multimedia Clients (IMC). A Primary station acts like a multimedia streaming source. The Antenna server keeps an association between the globally scoped multicast address and the locally scoped multicast address. Each local server is equipped with an advertisement server. The architectural details and description of each functional component of MarconiNet have been described in [10], [11].

In each cell (subnet) there is at least one local server (Radio Antenna Server) which converts globally scoped incoming multicast stream to a locally scoped multicast stream. This server can act like a router but it can also be a proxy by performing application layer routing. There can be multiple sources, where each multimedia stream delivery is associated with a unique globally scoped multicast address.

By introducing the local servers one can have full control on the program being broadcast, such as transmitting global program/local program, introducing local advertisement. Since these local servers act like affiliates, a payment scheme can be devised between the global station and local affiliates. The local commercial server is a part of the local affiliate which can be a streaming server and can be controlled by different protocols such as RTSP [12]. End clients can be SIP [13] enabled so as to be able to invite another user to a multicast session.

MarconiNet also promises to offer flexible streaming wireless services that deal with localized multicast while taking care of mobility of the clients. This paper mainly focuses on channel monitoring, payment scheme, mobility and QoS mechanism that are based on several application layer techniques such as SIP and RTCP [15] to control the stream delivery and mobility.

In order to take care of dynamic load balancing and handoffs, multiple local servers can be instituted within the same subnet or across the subnets in a domain, but then it needs a proper coordination between these servers for redirection of stream. This detailed discussion of multiple server interaction is for future study.

Figure 1 provides presents an overview of MarconiNet for streaming application, and figure 2 provides a logical architecture for the same.

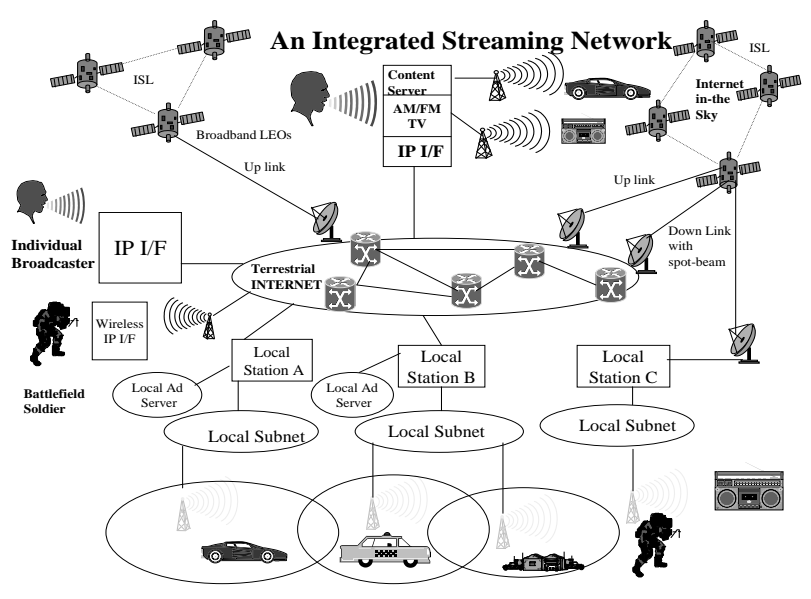

Fig. 1. MarconiNet for Streaming Application

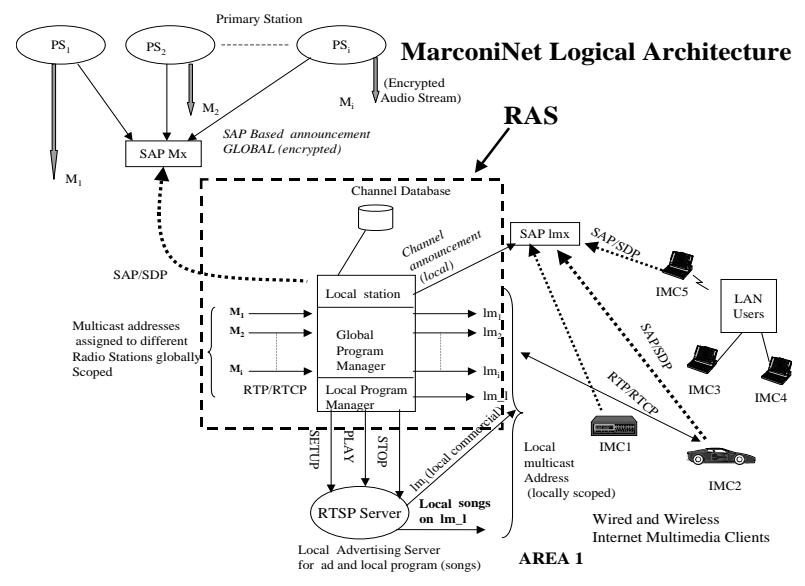

Fig. 2. MarconiNet Logical Architecture

\section{RELATED WORK}

The very process of joining/leaving a specific multicast group while changing the cell or subnet can be treated as equivalent to surfing a TV/Radio or flipping the channels, a typical behaviour found in a study among the adults. Details can be found in reference [16]. Mobility and stream delivery in MarconiNet take advantages of localized IP multicast techniques in a wireless environment which is equally applicable to Internet Radio/TV surfing.

There has been some previous work that discusses the group join/leave behavior in the Internet, effect of channel surfing, and mobility of multicast stream. Almeroth et al [17] have described the multicast group behaviour in the Internet, and have cited some results about surfing by looking at Mbone's temporal statistics. It shows that within a time interval of 2 minutes, a user leaving one session either joins another session or becomes inactive. Although this is very similar to a mobility model, where the user leaves one group and rejoins the same group in the next cell, that study has not taken into account the mobility of the users and the associated parameters. However references [18], [19] and [20] 
describe many of the architectural issues associated with mobile hosts in a multicast environment. A mechanism to obtain fast-handoff for unicast communication using localized multicast technique has been described in [21].

Also references [22], [23] propose ways for taking care of fast delivery of multicast stream when the end-hosts are mobile within a domain. Reference [22] proposes a solution of handover with pre-registration, in order to provide fast-handoff for the multicast streams while moving between subnets. This is accomplished by sending a unicast datagram to the neighboring station about the multicast address that it is subscribed to, so that the neighboring station would have joined the multicast tree even before the client moves in to the neighboring cell. This solution assumes there is an agent (Mobility Support Agent) in each subnet that invokes the join message.

Traditionally configuration time to obtain the network parameters and Internet Group Management Protocol(IGMP) [24] join/leave latencies are the most important factors that decide whether the client loses any transient packets or there is any waste of bandwidth because of flow of multicast stream in the previous cell.

Reference [25] provides a scheme to take care of loss of transient data for the mobile hosts by assigning location independent unique multicast address to each mobile host, so that there will be less transient data loss destined to the mobile host. However this scheme does not talk about the mobility of the localized multicast sessions.

Also [26] provides a mobility scheme for multicast sessions for wide area networking and adopts Mobile IP based approach to take care of mobility of multimedia traffic.

Most recently, Packet Video in conjunction with DoCoMo has started providing wireless multicast streaming services to the end users, but it has not taken into account the subnet mobility factor.

While all these approaches provide network layer solutions, this paper provides an application layer solution using real-time feedback mechanism based on RTCP, SDP [27], SAP [28] and SIP in MarconiNet environment.

\section{MarconiNet Business Model}

Channel monitoring, logging mechanism, payment model and security components associated with this architecture provide a novel business model for Mobile E-Commerce. All these components are based on RTCP based feedback mechanism associated with the streaming traffic which is RTP based.

For each channel being diverted by the local station, additional RTCP signaling channel is created with different port. Each listener sends RTCP SDES packets to notify the local station (RAS) alongwith the client's demographic information and the respective channel it is subscribed to. RAS maps each listener to the desired channel, which in turn increases the number of listeners for that particular channel. The listener-to-channel mapping is destroyed via RTCP BYE packets or via RTCP timeout feature. By doing this it also de- creases the number of listeners for the associated channel at the local station. Channel monitor provides a real-time view of how many clients are tuned to a particular channel at a particular point of time.

The logging mechanism has a very close ties with Ecommerce application and provides a very good way of generating revenue. The purpose of logging mechanism is to facilitate for advertisers the process of determining when and on which channels to place their commercials in order to maximize returns on investment. The higher level of advertising efficiency will drive more advertisers towards advertising on the Marconi server. This in turn will allow the Marconi server to pursue an increasing share of Internet Radio/TV advertising market by charging different prices for different segments of air-time, based on demand.

This reporting mechanism enables Marconi server operators to track user listening trends and can provide to potential/existing advertisers detailed reports with users' listening statistics. This concept relies heavily on the RTCP triggering model that is supported by Marconi. Even though the primary purpose of RTCP is to notify Marconi when users join a local multicast group to start listening on an audio channel, RTCP is also ideally suited for allowing Marconi to gather user-specific and channel specific information.

Since security is an important aspect of any E-commerce model, it is important to provide proper security association between the global station, local affiliate and end-clients. The proposed MarconiNet architecture presently offers four level of encryption overall. Global announcement encryption, global multicast stream encryption, local audit encryption and user authentication. By using global announcement encryption we can separate global announcements from the local announcements. The local clients should not be able to get access to the global announcements and would only be able to view the local announcements which is controlled by the server. By using a global encryption key during the announcement the global stations do not allow the Internet multimedia clients to find out about the global channels, rather it gives the the control over to the local stations to announce subset of these channels to the local clients. On the other hand security model for global multicst stream should effectively prevent the Internet Multimedia Clients, as well as the non-paid RASs (local affiliates) from receiving the broadcast content. Thus each radio/TV station (RSC) must maintain a secret key and encrypt all outgoing content so that only ciphertext stream is transmitted. The basic strategy is to generate a symmetric encryption key at the station and securely distribute this key to a particular RAS or local server upon its payment.

Global multicast stream encryption can be extended to local section as a second level hierarchy. Some of the pay-perlisten program are announced to the local multicast addresses in any domain using encryption key so that proper charging mehodology can be in place for the local clients. An RTCP based Multicast Group Access Control can be used, where an access token based on the host parameters can be sent as part 
of RTCP "JOIN" message before an end client is able to listen to the multicast traffic similar to IGMP authentication mechanism proposed in [29]. As the end client moves from one subnet/domain to another and associates itself to a different local server, it needs a mechanism to transfer the security association to the second server where the client is moving. A scheme similar to [30] can be instituted to distribute this key to the adjacent local server.

This will also help to preserve the sensitive information such as the secret keys (of RSCs, and pay-per-listen channels), user accounts, and payment data. Advertising companies can be authenticated so that un-authorized companies cannot try to play with the local Advertisement Insertion system. The security association between the global station and local affilate is taken care of as follows. Each local station generates its own PubKey/PrivKey pair. Each global station generates a SEK (Secured Encryption Key) and begins transmitting encrypted audio content. This SEK needs to be distributed to the participating local stations in a secure way so that other local stations that have not paid cannot obtain this key. Public key technology is employed for this purpose. The local station submits its public key to the primary station alongwith its payment or CAs (Certificate of Authority). Local station (affiliate) in turn receives an integer ID from the global station which is later used to index the SEK distribution list. The PS collects the public keys from the local stations and add these to its SEK distribution list upon their payment. This hierachical based security system will enable an ideal charging mechanism and payment model.

Based on the channel monitor, logging mechanism and hierarchical security structure, several kinds of payment models can be devised. Local stations (Radio Antenna Server) collect the fees from the local commercial companies so as to be able send the advertisement during the commercial break while relaying the global stations. Besides relaying the program from the common global stations, the local stations also relay some pay-per-listen program, in which case it pays to the global station depending upon how many listeners are listening to a particular program. This can be determined from the RTCP reports generated from the Internet Clients. Every local station also broadcats its local program to the local clients with segments of news or some other premium programs relayed from the global stations. Two kinds of payment model can be designed, there may be users who would like to listen to the program without being interrupted by the advertisement and some who would not really mind the local ads.

Since mobility and QoS are part of this streaming architecture, as the client moves around from one subnet to another, itis important to ensure that the client gets same quality of service. Some of the mechanism to ensure proper handoff and QoS guarantee are described later in this paper. As RTCP provides state of QoS on the client side, it will be easy to devise a QoS based pricing scheme.

\section{Mobility COMPOnEnTS}

In general, during a node movement, signaling and transport delays contribute to the latency of multimedia stream delivery associated with any two-party or multi-party communication session. Terminal mobility can be categorized as presession and mid-session. Pre-session mobility generally does not contribute to the delay associated with the on-going session, but may add delay to any new upcoming session. Signaling such as registering with a new server, notifying the communicating party with the mobile node's new contact address, inviting another user to a streaming session generally constitutes signaling delay, while transport delay dominates the delay component associated with the mid-session mobility.

Unicast stream delivery in a mobile environment could be supported either by a network layer solution such as one of the variants of Mobile IP or by an application layer solution such as SIP [31], [32]. Since it seems that SIP will eventually be part of the mobile Internet protocol architecture, it may make sense to leverage some of its inherently present mobility support functions. SIP can help provide personal mobility, terminal mobility, session mobility and service mobility. For the purposes of MarconiNet, in this paper, we will elaborate on the terminal mobility support for multicast, however a comprehensive discussion on other SIP based mobility types can be found in [33]

The application layer approach (specifically SIP) aims to keep mobility support independent of the underlying wireless technology and may fit better into the area which MarconiNet addresses, i.e. the provision of multimedia services for the mobile users.

Latency associated with receiving continuous unicast/multicast stream from the same source while the client moves to the next cell consists of several components such as detection of a new cell/subnet/domain $\left(\Delta_{1}\right)$, address acquisition and network configuration $\left(\Delta_{2}\right)$, triggering of multimedia stream to be delivered in the new subnet $\left(\Delta_{3}\right)$ and actual delivery of multimedia stream $\left(\Delta_{4}\right)$. While some of these factors are common to both unicast and multicast stream delivery (e.g., cell/subnet detection, IP parameter configuration), we concentrate mostly on delivery schemes for multicast streaming traffic in this paper.

For a mobile node in MarconiNet environment, latency is composite of actual hand-off time and join interval. Handoff delay includes the time to detect that it is in a new cell/subnet/domain, time for obtaining an IP address from a DHCP[34] or DRCP [35] server if it is moving between the subnets, and some triggering mechanism that will help initiate the multimedia flow in the new location. This would decide the join/leave latency factors of the multimedia flow.

Figure 3 shows a basic diagram of call flow with standard sequence of events for micro and macro mobility, and in next section these steps are discussed in details. In this diagram some of the interim message flows (e.g., DHCP ACK etc.) are omitted for brevity. For domain mobility there are other 


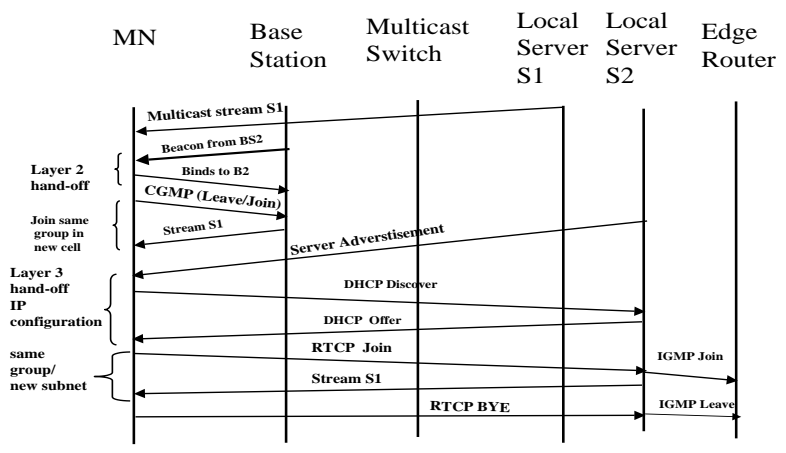

Fig. 3. A handoff flow

factors such as AAA (Accounting, Authentication and Authorization) that plays a big role in deciding the delay factor for media delivery, because of the steps for establishing security association between the local servers in each domain, especially the steps involved in authentication and creating local security association.

\section{A. Movement Detection}

As the mobile moves away from its own cell, and begins to move to a new cell, it would likely to receive the stream from a new local Marconi server in the adjacent cell.

However in some cases in order to provide proper load balancing, there may be two local servers in the same cell served by the same base station. In that case load balancing needs to be provisioned to make sure that the client is achieving proper QoS requirement by switching to the proper server.

Discovering a new cell/subnet/domain can be realized in different layers. During mobile's handoff, first movement detection takes place in layer 2 , where the client would decide to switch over to a new base station based on the signal strength. In the case of CDMA, soft handoff is initiated, so that each client can listen to both the base stations, and receive both the stream, and then it decides as to which one would be accepted from the mixed signal. As soon as it switches over to a new base station and layer two handoff is over, it would need to figure out if the client is in a new subnet or domain altogether. Using layer 3 triggering mechanism such as router advertisement method similar to ICMP router advertisement in Mobile IP it can be determined if the client is in a different subnet. An application layer detection mechanism such as server advertisement method can as well be used, if the client is involved in a real-time communication session. However it may be faster to achieve the handoff notification using layer 2 mechanism, although experiments show that application layer techniques provide flexibility. Some of the atomic functions are described in more detail in the following subsections.

\section{B. Network configuration}

In the cases where Mobile IP's Foreign Agent approach is not instituted, as the client moves from one cell to another, where the new cell is another subnet it would either obtain a new address from the DHCP/DRCP server or can use standard Mobile IP approach to obtain a new care-of-address from the foreign agent while keeping its own IP address unchanged. There are other approaches such as cellular IP [36], HAWAII [37] for intra-domain mobility where IP address does not change once it enters a new domain. But some of these use source based routing that adds more complexity and overhead to the routers within a domain.

In case of subnet movement typical time for acquiring a DHCP address would be about 5-10 seconds [38], although there are various other alternatives to take care of this problem such as DRCP[35] which is a light-weight version of DHCP suitable for wireless roaming environment. Using DRCP the address acquisition takes place within a few hundred milliseconds. This timing can be compared with the auto-configuration in DHCPv6.

Although subnet detection and address acquisition are important factors for the delay associated with multimedia stream delivery, focus of this paper is about the multicast session, join and leave latency mostly.

\section{Triggering Multimedia stream}

In order to maintain minimum loss and latency during the client's movement it would be desirable to minimize the handoff time and to provide almost instantaneous flow of multicast stream by adopting some novel triggering mechanism. Similarly it may be required to avoid the waste of bandwidth because of continuity of flow of traffic associated with the leave latency.

Triggering technique of multimedia stream can be instituted in several layers such as layer 2, layer 3 and application layer.

In traditional layer 3 method, triggering delay would consist of IGMP query report after the node moves to the new cell so as to be part of the same multicast tree. But if there is currently at least another active participant in the subnet, the mobile host can continue to receive the traffic without waiting to hear the membership query from the router. A typical query interval for the IGMP is by default 125 seconds [39], although this value is configurable in the multicast routers. In order to avoid the flooding in the LAN with IGMP messages this value cannot be made very small. Reference [40] shows that by using IGMP, a host will wait for 65 seconds on average in order to continue to receive the multicast traffic after the hand-over. This is because IGMP was not designed for roaming clients in a wireless environment where handoff latency and packet loss are important concerns. Similarly a typical leave latency once the host has moved to a new subnet is about 2 mins, i.e. traffic would still flow to the previous cell even after the client has moved out, thus wasting bandwidth in the previous cell.

If the client is moving within a subnet but between the cells, it does not have to spend time in obtaining a new IP address, but rather handoff is taken care of at layer 2 with the help of 
co-ordination between the access points. In this case the triggering of multimedia stream can be taken care of by a variation of Generalized Switch Management Protocol [41] such as CGMP(Cisco Group Management Protocol)[39] or a variation of IGMP snooping [39] can be instituted at subnet. This method is a typical variation of layer 2 multicast. It would allow the multicast traffic to be filtered out at the multicast switch so as to save valuable wireless bandwidth when there is no active participant in the adjacent cell which is being served by the same switch.

\section{MarconiNet Stream Delivery Techniques}

Multimedia stream delivery during a node's movement can be achieved by deploying localized multicast techniques at different layers of the multimedia protocol stack.

In MarconiNet environment, the mobile clients use locally scoped common multicast address for indexing and use a locally scoped multicast address to tune to a particular stream from a source. Although global multicast address remains the same, there is a likelihood that the local multicast address on each of the server's local interface would be different. Thus mapping association between global and local address is stored locally in Radio Antenna Server's database.

Figure 4 shows a typical address assignment where $I_{a}, I_{b}$, $I_{c}$ are the globally known subnets connected to one of the interfaces of the servers where as $i_{a}, i_{b}, i_{c}$ are the local subnets connected to the secondary interfaces of the servers and could be local. According to the MarconiNet model, server receives the multicast stream through its global interface and redirects it out through the local interface for the local clients in each cell. In this particular picture, S1, S2 ... S5 are local stations (servers) connected to the upstream routers. Each server (with the exception of S2 and S3) is connected to a different subnet and has a separate interface. S2 and S3 are connected to the same subnet via a multicast switch capable of taking care of layer 2 multicast. Each station serves one cellular region underneath, which could be part of a private subnet dedicated for the local users.

In order to make sure that each moving client keeps on pointing to the same multicast address or belong to the same group membership, a mechanism would be necessary to preprovision the locally scoped multicast address. It can be quite possible that the mapping between the global and local address association is determined by a policy decided at the local station/Antenna Server.

In order to make the mapping more efficient, there can be a rule based association between the global address and local address, say using an offset of $\mathrm{N}$ on each octet (at least between the servers on the same domain). Otherwise if the mapping is different at each local server, a proactive mechanism is needed to inform the adjacent server about the local address for the stream delivery, before the actual stream gets delivered.

Figure 5 shows an architecture illustrating stream redirection to the wireless multicast clients by introducing mul-

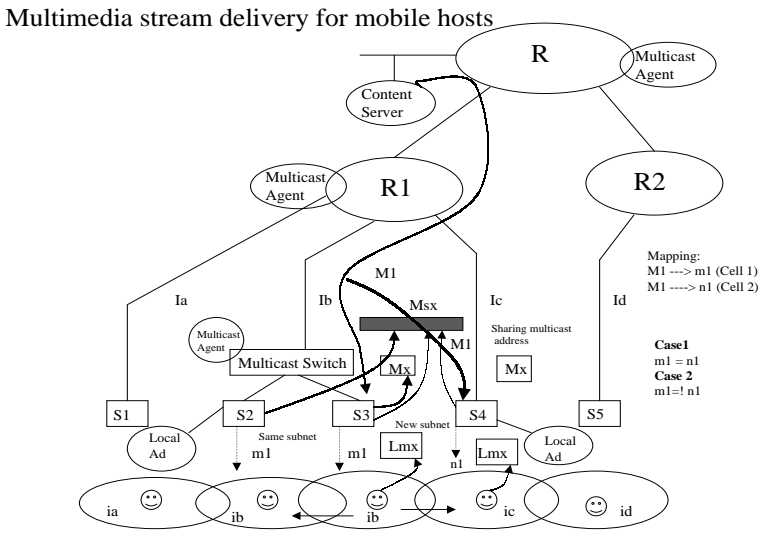

Fig. 4. MarconiNet Fast-handoff scenario

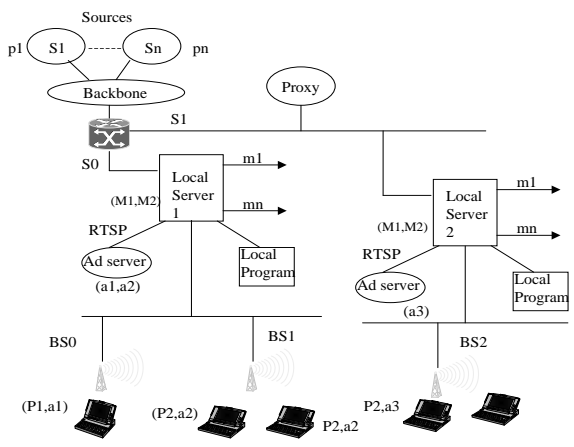

Fig. 5. Stream redirection with multiple servers

tiple local servers for MarconiNet. These servers can be widely distributed to provide a hierarchical structure, but are installed at the edges of the network to provide localized services using locally scoped multicast.

Following subsections provide several methods associated with stream delivery at different layers such as layer 2, network layer and application layer.A fast delivery mechanism of multicast streaming is intended here to avoid the loss of realtime data and improve the latency. These could be achieved in several different ways as described below.

\section{A. Layer two multicast}

Multicast technique can be used at several layers to provide flexible services. Layer 2 methods come into picture when the adjacent cell where the client is moving to belongs to the same subnet. Several methods such as IGMP snooping, Cisco Group Management Protocol (CGMP) and IEEE 802.1 p can be used to take care of layer 2 handoff for the multicast streams.

In cases where the destination cell belongs to the same subnet and both the cells are served by the same local server, 
then multicast stream flows in both cells in the absence of any multicast switch. Although this would save the triggering time, it would however contribute to the waste of bandwidth, if there is no active participant in the adjacent cell. However it would be a trade-off between the triggering time and bandwidth saved. Typically as soon as the change of base station is detected, CGMP Join/Leave is triggered thus starting the flow of a multicast stream or keeping it from flowing to the next cell.

When the client moves between the cells which do not belong to the same subnet, there are several ways stream delivery and re-direction can be obtained to reduce the transient loss using layer 3 and layer 4 techniques. Some of these techniques are described in later sections. In general movement between the subnets may involve dynamic IP address assignment as the client moves around. However it is important to note that a new IP address assignment is not always needed for multicast delivery.

\section{B. Post-Registration}

Post-registration approach is the most common method but it takes the maximum time for the same multicast stream to get directed to the new cell. In a typical scenario, the client moves to a new cell, obtains the new IP address if the new cell is in a new subnet (it is not always necessary to obtain a new IP address while moving between subnets for multicast stream delivery), and then sends the join query via IGMP method. In these cases IGMP could be modified to provide aggregate group report, as suggested in [42].

Here we propose a new approach that would use an application layer triggering mechanism such as RTCP as in reference [10] in order to facilitate the join and leave of the session. This approach proposes an integrated solution of using RTCP and IGMP. Using an RTCP based triggering offers a solution at the user space (Experimental results of an analysis of advantage of using either of the methods are being investigated currently). In this case the end-client that is listening to a local multicast stream would send the triggering signal by sending RTCP JOIN/BYE using the SDES field of RTCP. In this scenario, triggering at the lower hierarchy would be accomplished by RTCP, but the local server would trigger the multicast flow from the upstream router using IGMP method.

\section{Pre-registration Method}

Each local station (server) can have multiple neighboring (servers) stations. For each of these stations sharing an overlapping area with another station there would be a multicast announcement (address) associated that would be preprovisioned, where each station can point to find out the program subscribed to (group address) by the impending mobile host. Just before a mobile node leaves (decides to leave) the cell, a decision that could be based on the threshold value of the layer 2 signal, or some other soft handoff methodology as described in CDMA, it sends an RTCP message to the local announcement address, the server in turn would announce that to the sharing multicast addresses where the neighboring stations would be listening to in the global space. The neighboring stations (servers) look up to the multicast address and check it with their own databases to see if this multicast stream is already subscribed to. If this is the case, since other clients have been listening to the same stream, then it does not do anything, if the stream is not playing then the server sends an IGMP message to the upstream router and passes the stream to the local cells using a locally scoped multicast address, even before the client has moved to the new cell. Thus a soft hand-off is emulated for the associated stream. As soon as the client moves into the next cell it can still get the same stream without any interruption. The only tradeoff with this scheme is that bandwidth would be wasted for sometime because the multicast stream would be playing beforehand. Similarly the client would send an RTCP BYE to the server as it moves away from the previous server. This approach is better than the approach described in [22] where the client has to send a unicast packet to the neighboring station, that means the client has to know the addresses of the neighboring servers beforehand.

\section{Pre-registration with Multicast Agent}

This section defines the use of a Multicast Agent to take care of the multicast streams. With this method there would be a multicast agent within each router which would forward the global stream to the respective global multicast addresses (e.g., for areas where these clients are trying to move) in each subnet for a specific period of time, so that each neighboring server would receive the stream irrespective of whether the mobile node is moving into that cell or not. As soon as the mobile node moves into the new cell it sends an RTCP signal to alert that the mobile node has moved in, so the respective timer does not need to get triggered. However this approach would create waste of bandwidth in each of these subnets for a period of time.

\section{E. During the Registration}

In another approach, group association information can be passed on as a part of registration to the new network. When the node moves in, and tries to acquire the IP address in the local subnet it can send the request for that particular stream in its DHCP Discover option message about the address it has been listening to. But in that case the server would have to be a registration server. At the time of obtaining the IP address from the DHCP server, the client can send the locally scoped multicast address to the server, and depending on whether the server is already part of the multicast tree, it would ignore this request or re-join the tree. It remains to be seen if the client can use RTCP before the client obtains a DHCP address in the new domain.

\section{F. Proxy Registration}

Another approach would be to deploy proxy agents in each subnet. These proxy agents would join the upstream multicast 
tree on behalf of the servers, even before the clients move into the cell. The neighboring proxy servers would then be listening to a common multicast address to figure out the impending host's subscribed multicast address. In this case the multicast proxy would be sending the IGMP query messages beforehand on behalf of the local servers. This is a typical case where layer 3 multicasting technique has been used for the proxy servers.

\section{MARCONINET QOS APPROACH}

It is also of paramount importance that a mobile which is part of a multicast communication should be able to receive the same quality of service as it moves across subnets within a domain. We propose a combinatorial approach of diffserv technique [43] and application layer triggering technique based on RTCP feedback using RTCP RR (Receiver Record) and SDES. Reference [44] provides a diffserv based approach for unicast communication for mobiles within a domain. Our approach uses diffserv based technique by instituting policy control [45], [46] in the edge routers/servers, but relies on the RTCP feedback report to maintain QoS parameters for the multicast streaming traffic. As part of its RTCP feedback, each mobile would keep on advertising its QoS parameters (e.g., delay, jitter) on the locally scoped multicast address using SAP [28] and an extension of SDP.

This in turn would be advertised in the globally scoped multicast address (M) where both the adjacent servers can determine the QoS parameters for each mobile impending to move. If a mobile listening to a specific multicast address happens to be the first client in the adjacent cell, then it would need to make a reservation apriori so as to receive the desired quality of service for the streaming traffic as soon as it moves in there. In the testbed, since Marconi servers are Linux based routers, Linux's traffic control (tc) mechanism was used to set up the traffic priority destined for a particular multicast address in one of its interfaces. If a mobile client is not the first one entering to the new cell, QoS may not be an issue. While within a cell the client can also negotiate with the Linux server to control the traffic rate by making additional real-time request.

The incoming traffic can be conditioned at the server or at the upstream router. A receiver based adaptation scheme such as layered encoding scheme and multiple multicast groups for one session [47] can be used to take care of receivers with different QoS requirement. Figure 6 shows a realization of this real-time QoS management as the client moves from one subnet to another. This shows the mechanism as to how QoS would be maintained when the mobile moves from one subnet to another by using RTCP feedback. This QoS manager which implements Linux's traffic control mechanism can reside in each local server in the network that interacts with each other by means of the global multicast address.

Figure 7 shows a full blown IEEE 802.11b based integrated testbed, where mobility and QoS part of MarconiNet are being experimented currently. This testbed provides both indoor

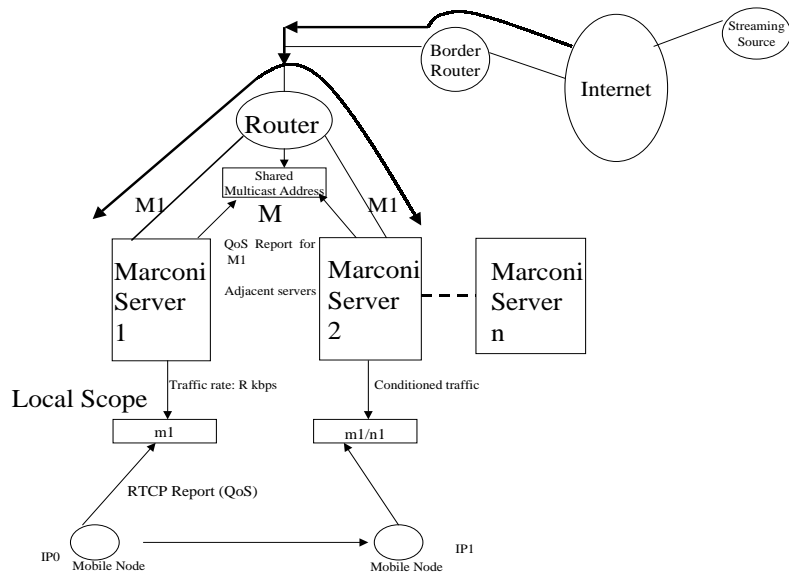

Fig. 6. MarconiNet Quality of Service

and outdoor mobility functionality, and emulates a wireless Internet by instituting cross traffic generator and delay simulator. In this testbed all three kinds of mobility scenario namely micro, macro and domain are being experimented. The edge routers are Linux systems running "mrouted". Basic measurements such as IGMP Join/Leave latency were taken when client moved around between the cells. Experiments were done for both the cases when the client changed its IP address and when it did not. As part of the initial experiment many of the multimedia streaming applications were used using some of the Mbone tools such as sdr, rat, vic, wb and MarconiNet application. Several tunnling techniques such as UMTP were used to demonstrate the streaming application in the event of non-availability of multicast routers in some parts of the network. Application layer triggering methodology is being implemented to provide a faster join and leave latency. Each of the servers has a media database for local advertisement. In addition to be able to explore faster stream delivery methods for different kinds of mobility scenario, other aspects of MarconiNet such as QoS management based on real-time feedback of streaming content, and different location based services such as localized advertisement insertion, inviting another client to a multicast stream using SIP are being realized.

\section{CONCLUSIONS}

In this paper we discussed a business model viable for M-commerce based on MarconiNet architecture that can be applied to many commercial applications including Telematics. Focus of this paper has been the operational techniques needed to build this model that include secured content distribution, payment structure, faster join and leave latency for the clients using application layer approaches, timely delivery of multimedia content for the moving clients and maintaining the QoS in wireless multicast environment. It discusses many of the latency factors associated with streaming multimedia in MarconiNet and presented several scenarios for obtaining fast handoff using localized wireless Multicast. As part of fu- 


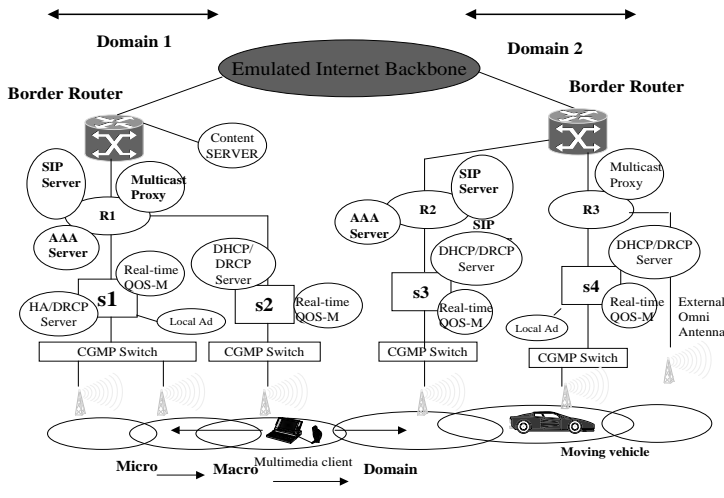

Fig. 7. MarconiNet Realization in an Integrated Testbed ture work we plan to integrate SIP with RTSP server such as inviting an RTSP media server using SIP signaling, or inviting another SIP enabled receiver to an ongoing multimedia conference and coordination between the neighboring local servers for proper load balancing and media delivery.

\section{REFERENCES}

[1] D Estrin et al, "Protocol Independent Multicast (PIM) Dense Mode"

[2] J. Moy, "Multicast Routing Extension for OSPF, "Communication of the ACM"

[3] D. Waitzman, C. Patridge, S. Deering, "DVMRP (Distance Vector Multicast Routing for the Mbone", RFC 1075, November 1988.

[4] A. Ballardie, P. Francis, J. Crowcroft, "Core Based Tree(CBT)", "Proceedings of ACM SIGCOMM.

[5] Border Gateway Multicast Protocol, "draft-ietf-bgmp-spec-02.txt", Internet Draft, Work in Progress, November 2000

[6] Source Specific Multicast, www.ietf.org (SSM working group)

[7] UDP Multicast Tunneling Protocol, draft-finlayson-umtp-06.txt, IETF Workin Progress.

[8] D. Thaler et al., IPv4 automatic Multicast without Explicit Tunnels (AMT), IETF draft-ietf-mboned-auto-multicast-00.txt, February 2001, work in Progress

[9] Bob Quinn, Kevin Almeroth, "IP Multicast Applications: Challenges and Solutions", draft-ietf-mboned-mcast-apps-02.txt, IETF Work in Progress

[10] A. Dutta, H. Schulzrinne and Yechiam Yemini, "MarconiNet - An Architecture for Internet Radio and TV Networks" in Proc. of NOSSDAV 1999

[11] A. Dutta, H. Schulzrinne, "A Streaming Architecture for Next Generation Internet" in In the proceedings of ICC 2001

[12] H. Schulzrinne, A. Rao, and R. Lanphier, "Real time streaming protocol (RTSP)," Request for Comments (Proposed Standard) 2326, Internet Engineering Task Force, Apr. 1998.

[13] Mark Handley, Eve Schooler, H. Schulzrinne, Jonathan Rosenberg, "Session Initiation Protocol", RFC 2543

[14] Perkins et al., "IP Mobility Support", Request for Comments (Proposed Standard) 2002, Internet Engineering Task Force, October 1996

[15] H. Schulzrinne, S. Casner, R. Frederick, and V. Jacobson, "RTP: a transport protocol for real-time applications," Request for Comments (Proposed Standard) 1889, Internet Engineering Task Force, Jan. 1996

[16] D.A. Ferguson, "Measurement of mundane TV behaviors: Remote control device flipping frequency", Journal of Broadcasting Electronic Media, 38(1), 35-47

[17] Kevin Almeroth, Mostafa Ammar "Multicast Group Behaviour in the Internet's Multicast Backbone (MBone)", IEEE Communication Magazine, June 1997.

[18] G. Xylomenos and G.C Polyzos, "IP Multicast for Mobile Hosts", IEEE Communications Magazine, January 1997

[19] Upkar Varshney and Samir Chatterji, "Architectural Issues to support multicasting over wireless and Mobile networks" 1998 WCNC
[20] A. Acharya, A. Bakre, B. Badrinath, "IP multicast extensions for mobile internetworking, in Proc. IEEE INFOCOM'96, SF, CA.

[21] A. Misra, S. Das, A. Dutta, A. McAuley, "IDMP-based Fast Handoffs and Paging in IP-based cellular networks" In the proceedings of 3G Wireless 2001.

[22] Jiang Wu, "An IP mobility support for 4GW wireless Infrastructure" 1999 Personal Computing and Communication Workshop (PCC Workshop 99), November 1999

[23] A.J. McAuley et al, "Mobile Multicast Proxy" in the Proceedings of the IEEE MILCOM Conference 1999, OCtober 1999

[24] W. Fenner, "Internet group management protocol, version 2," Request for Comments (Proposed Standard) 2236, Internet Engineering Task Force, Nov. 1997.

[25] J. Mysore, V. Bharghavan, "A New Multicasting-based Architecture for Internet Host Mobility" Proceedings of Mobicom 1997

[26] Vineet Chikarmane, Carey L. Williamson et al, "Multicast support for mobile hosts using Mobile IP: Design Issues and Proposed Architecture" Mobile Networks and Applications

[27] M. Handley and V. Jacobson, "SDP: session description protocol," Request for Comments (Proposed Standard) 2327, Internet Engineering Task Force, Apr. 1998.

[28] M. Handley, “SAP: Session announcement protocol,” RFC 2974

[29] www.securemulticast.org

[30] Iolus: A Framework for Scalable Secure Multicasting, SIGCOMM 1997

[31] Elin Wedlund, H. Schulzrinne , "Mobility Suport using SIP" in IEEE/ACM Multimedia conference WOWMOM 1999

[32] A.Dutta, F. Vakil, J.C Chen, S. Baba, N. Nakajima, H, Schulzrinne, "Application Layer Mobility Management Scheme for Wireless Internet" In the proceedings of 3G Wireless 2001 conference, San Francisco.

[33] H. Schulzrinne and E. Wedlund, "Application-layer mobility using SIP", ACM Mobile Computing and Communications Review, vol.4, no.3, July 2000.

[34] R. Droms "Dynamic Host Configuration Protocol" Request for Comments (Proposed Standard) 2131, Internet Engineering Task Force, March 1997

[35] A.J. Mcauley, S. Das et al, "Dynamic Registration and Configuration Protocol" IETF Draft, work in progress.

[36] Andrew T. Cambell, Javier Gomez, Andras Valko, “ An overview of Cellular IP" IEEE Wireless Communications and Networking Conference, September 1999.

[37] R. Ramjee, T. La Porta et al, "HAWAII: A Domain-based Approach for Supporting Mobility in WIde-area Wireless networks, in the Proceedings of 7th IEEE International Conference on Network Protocols, 1999(ICNP'99), OCtober 1999

[38] J-O. Vatn and G.Q. Maguire Jr., "The effect of using co-located careof addresses on macro handover delay, in the Proceedings of the 14th Nordic Tele-traffic Seminar (NTS 14), August 1998

[39] B. Williamson,'Developing IP Multicast Networks: The Definitive Guide to Designing and Deploying CISCO IP Multicast Networks, Volume I, January 2000, Cisco Press".

[40] M. Flament et. al, "An approach to 4th Generation Wireless Infrastructure- Scenarios and Key Research Issues". VTC 99, May 1999

[41] Avri Doria, Kenneth Sundell, "draft-ietf-gsmp-08.txt", General Switch Management Protocol V3, IETF work in Progress

[42] Satwant Kaur et al, "Multicast Support for mobile IP using a modified IGMP" in the Proceedings of the IEEE Wireless Communications and Networking Conference, 1999 (IEEE WCNC'99), September 1999.

[43] IETF, Differentiated Services (diffserv) working group. http://www.ietf.org/html.charters/diffserv-charter.html

[44] J.C Chen et al, "Dynamic SLS Negotiation Protocol" 2000 Workshop on Quality of Service, Las Vegas

[45] Werner Almesberger, "Linux Traffic Control - Implementation Overview"

[46] Saravanan Radhakrishnan, "Linux - Advanced Networking Overview", Technical Report, University of Kansas.

[47] Steve McCanne, Van Jacobson, Martin Vetterli, "Receiver Driven Layered Multicast", ACM SIGCOMM'96, August 1996.

[48] T. Friedman and D. Towsley, "Multicast session membership size estimation," in Proceedings of the Conference on Computer Communications (IEEE Infocom), (New York), Mar. 1999. 